\title{
Investigating the Influence of a Compact's Geometry on Its Pore Structure and Optical Properties Using Terahertz Spectroscopy
}

\author{
P. Bawuah, ${ }^{1}$ R. Dong, ${ }^{1}$ M. Al-Sharabi, ${ }^{1}$ D. Markl ${ }^{2,3}$ and J.A. Zeitler ${ }^{1}$ \\ ${ }^{1}$ University of Cambridge, Department of Chemical Engineering and Biotechnology, UK, \\ ${ }^{2}$ University of Strathclyde, Strathclyde Institute of Pharmacy and Biomedical Sciences, Glasgow, UK \\ ${ }^{3}$ EPSRC Future Manufacturing Research Hub for Continuous Manufacturing and Advanced Crystallisation \\ (CMAC), University of Strathclyde, Technology and Innovation Centre, Glasgow, UK
}

\begin{abstract}
In this study, terahertz time domain spectroscopy (THz-TDS) is used to analyze how the geometry of a compact affects its pore structure (pore shape and orientation). By using flat-faced and biconvex compacts, it was evident from our analysis that pores tend to assume specific shapes and orientations based on the compact's geometry and this was found to significantly affect the extracted optical properties of samples prepared by mixing a material with polyethylene (PE) or polytetrafluoroethylene (PTFE) ethylene as diluent and subsequent compaction. However, such sensitivity to the pore properties opens a number of industrial applications such as for quality testing of pharmaceutical tablets.
\end{abstract}

\section{INTRODUCTION}

$\mathrm{R}$ ECENT studies have shown that THz-TDS coupled with Bruggeman's effective medium theory is a powerful tool for measuring the pore structure of porous materials [1-2]. The pore structure, i.e. shape and orientation, significantly contributes to the mechanical and disintegration properties, but also directly influences the optical properties of the powder compact.

This study employs a similar THz-TDS method to investigate how the geometry of a porous compact influences the overall pore structure and hence the refractive index of the compact. This was possible by, firstly, measuring the effective refractive index of the compacts from reference and sample terahertz pulse transmission measurements and then adopting the anisotropic Bruggeman effective medium approximation (ABEMA) [2].

The AB-EMA model allows the estimation of a shape parameter, $L$, by using an iterative data fitting technique. By assuming a spheroid with unique axis $a$ and two common axes $b=c$ [2], the $L$ factor is related to the aspect ratio of the pore as

$L=\frac{1}{1+1.6(a: b)+0.4(a: b)^{2}}$

Using an HB50 compaction simulator (Huxley Bertram Engineering Ltd., Cambridge, UK), several batches of flatfaced and biconvex porous compacts with a similar porosity range were compacted.

Terahertz time-domain measurements of all the batches were conducted using a TeraPulse 4000 spectrometer (TeraView Ltd., Cambridge, UK). The intrinsic refractive index as well as the pore shape of the tablets were extracted from the measured terahertz effective refractive index using the AB-EMA [2].

\section{REsUlts}

Despite the same material composition, as well as the similar porosity range of the porous compacts, our measurements indicate that the flat-faced and biconvex compacts possess different pore geometries (see Fig. 1). This directly affects the values of the extracted optical properties. The effective refractive index and solid refractive index of the flat-faced compacts were lower than that of the biconvex compacts.

An exhaustive study, based on the use of PE and PTFE pellets that are compacted under various controlled conditions, is currently ongoing with the aim of seeking a thorough understanding on how compaction parameters affect terahertz measurement for typical sample pellets.
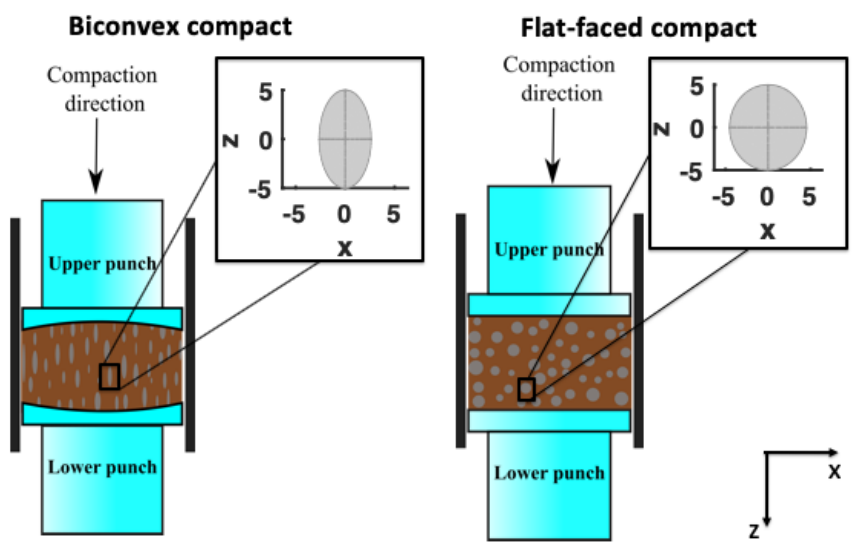

Fig. 1. Estimated pore shape of the biconvex and flat-face compacts using the calculated $L$ factor.

\section{SUMMARY}

This study highlights that the preparation of sample pellets has considerable effect on the optical properties that are extracted from typical THz-TDS measurements. It complements previous work to guide the community how to acquire the best quality spectral data $[3,4]$.

\section{REFERENCES}

[1] D. Markl, P. Wang, C. Ridgway, A.-P. Karttunen, P. Bawuah, J. Ketolainen, P. Gane, K.-E. Peiponen, J.A. Zeitler, "Resolving the rapid water absorption of porous functionalised calcium carbonate powder compacts by terahertz pulsed imaging," Chem. Eng. Res. Des. $132,1082-$ 1090, 2018.

[2] D. Markl, P. Wang, C. Ridgway, A.-P. Karttunen, M. Chakraborty, P. Bawuah, P. Pääkkönen, P. Gane, J. Ketolainen, K.-E. Peiponen, J.A. Zeitler, "Characterization of the Pore Structure of Functionalized Calcium Carbonate Tablets by Terahertz Time-Domain Spectroscopy and X-Ray Computed Microtomography," J. Pharm. Sci. 106, 1586-1595, 2017.

[3] P.U. Jepsen and B.M. Fischer, "Dynamic range in terahertz time-domain transmission and reflection spectroscopy," Opt. Lett. 30, 29-31, 2005.

[4] M. Frenz, B.M. Fischer and M. Walther, "The Christiansen effect in terahertz time-domain spectra of coarse-grained powders," Appl. Phys. Lett. 92, 021107, 2008. 